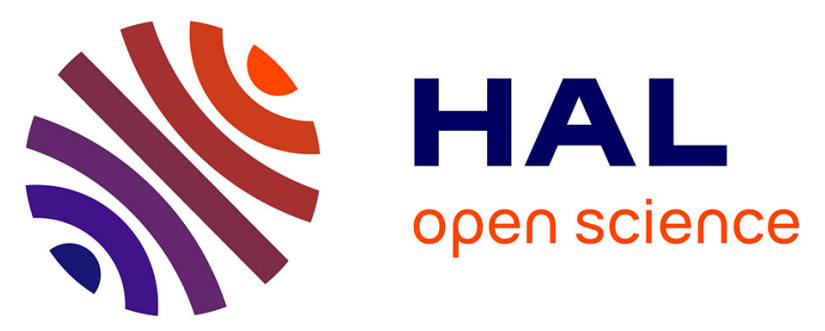

\title{
On Designing Sensor Networks with Smart Antennas
}

Ghalem Boudour, Aubin Lecointre, Pascal Berthou, Daniela Dragomirescu, Thierry Gayraud

\section{To cite this version:}

Ghalem Boudour, Aubin Lecointre, Pascal Berthou, Daniela Dragomirescu, Thierry Gayraud. On Designing Sensor Networks with Smart Antennas. 7th IFAC International Conference on Fieldbuses and nETworks in industrial and embedded systems, Nov 2007, Toulouse, France. p. 349-356. hal00356852

\section{HAL Id: hal-00356852 https://hal.science/hal-00356852}

Submitted on 28 Jan 2009

HAL is a multi-disciplinary open access archive for the deposit and dissemination of scientific research documents, whether they are published or not. The documents may come from teaching and research institutions in France or abroad, or from public or private research centers.
L'archive ouverte pluridisciplinaire HAL, est destinée au dépôt et à la diffusion de documents scientifiques de niveau recherche, publiés ou non, émanant des établissements d'enseignement et de recherche français ou étrangers, des laboratoires publics ou privés. 


\title{
On designing sensor networks with smart antennas
}

\author{
Ghalem Boudour, Aubin Lecointre, Pascal Berthou, Daniela Dragomirecu and Thierry Gayraud
}

\author{
LAAS-CNRS \\ University of Toulouse \\ Toulouse, France \\ \{prenom.nom\}@1aas.fr
}

\begin{abstract}
Wireless sensor networks (WSN) is an active research area. The recent advances in the fields of antennae and transmission lets consider massive improvements in the energy and communication management of WSN. However, the new WSN architectures have to evolve to take it into account. This paper presents an extension of the IEEE 802.15.4 protocol to take benefits of smart antennas technology. NS-2 simulations show the feasibility and the benefits of such architecture.
\end{abstract}

\section{INTRODUCTION}

Wireless sensor networks will be an important issue in our life in the near future. Driven by the development in wireless networking, Micro-ElectroMechanical Systems (MEMS), embedded processing, sensor networks are becoming attracting solutions for numerous applications (environmental monitoring, industrial sensing and diagnostics, military applications...). The European Commission foresees 100 times more communicating objects in the next ten years. Such an increase needs an important evolution in the design of communicating sensors nodes and in the network architectures.

The Defense Advanced Research Projects Agency (DARPA), manages and directs selected basic and applied research and development projects for the Department of Defense (DoD) and has funded several projects about low power wireless sensors networks. Several universities have participated to such projects: Stanford University, Bekerley (SMART DUST, PicoRadio group), University of California at Los Angeles (WINS), Rutgers University (WEBDUST), University of South California (PASTA) or Massachusetts Institute of Technology ( $\mu$-amps). Those projects focused on innovative energy-optimized solutions vertically across the protocol stack for different applications. For instance, PASTA (Power Aware Sensing Tracking and Analysis) investigates unattended ground sensor (UGS) applications and WEBDUST location dependent queries for WEB application.

The interest towards sensor networks led in 2003 to the definition of the IEEE 802.15.4 [12] standard proposed by the IEEE 802.15.4a Task Group. The 802.15.4 standard also forms the basis of the ZigBee [13] technology that provides a comprehensive solution for low data rate networking, from physical layer to applications.

Among the 802.15.4 drawbacks, we could quote the limited emission power level and the use of a saturated frequency band. Because of the fact the chosen band uses the $2,4 \mathrm{GHz}$ band. These latter is used by most of well-known communication protocols, such as Wifi. Bluetooth, etc...

In this paper we propose an extension to the 802.15.4 protocol that use directional antennas to mitigate these drawbacks. This architecture relies on recent attractive solutions for sensor networks. In particular, Ultra Wide Band (UWB) technique, use of millimeter wave bands and especially smart antennas that are important issues for future wireless local area networks, high rate ad hoc networks or sensor networks.

\section{DIRECTIONNAL ANTENNAS}

\section{A. Smart Antennas \& Energy Saving}

In the WSN literature, isotropic antennas are most of the time considered. The recent advance in transmission and the millimeter band allows using directional antennas because of the wavelength dependence of circuitry size. Figure 1 compares isotropic to directional antennas, also called smart antennas. Indeed, because of their small size, several small directional antennas can be integrated to a sensor instead a unique isotropic one.

Directional antennas have a bigger gain than classical isotropic antennas, since the beam width is limited. Consequently, the emission power could be reduced, thanks to the high gain of directional antenna. It is important in WSN context with low power constraint.

Besides, use power control [10] with directional antenna allows improving the low power capacity of our solution. Indeed, as described in figure 2, the addition of these two mechanisms offers a better energy ratio between energy used for transmission and energy used for reaching the considered receiver [1].

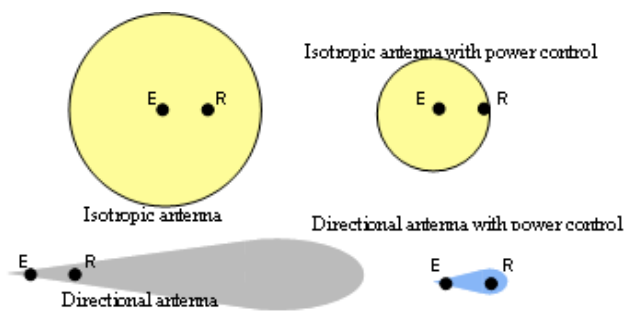

Figure 1. Illustration of the gain when using directional antenna with power control. 
Moreover, as the signal is emitted in a cone, instead of a sphere, the ISI is reduced. Indeed, ISI depends of delay spread and this latter will be lower in this case since the delay between the different way through the directional antenna is lower. The lower ISI is, the lower interference there is. Thus, the number of collision decrease also and consequently the number of retransmission. Consequently, the energy for correctly transmitting information decreases also (WSN power constraint). Decreasing ISI implies also a diminution of the complexity, and a higher data rate capacity.

Furthermore, in comparison with isotropic antennas, in this case, the network capacity is increased [17], by permitting to have distinct transmission in a small area without collision. It would be not possible with isotropic area because the emitted signal is over a large area (Figure 2). Figure 2 illustrates perfectly that the two links "E1 $\rightarrow$ R1" and "E2 $\rightarrow$ R2", are only possible with directional antennas. Indeed, with isotropic antenna only the link "E1 $\rightarrow \mathrm{R} 1$ " will be possible without generating collision.

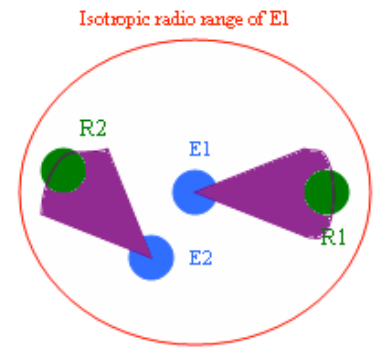

Figure 2. The increase of the network capacity thanks to directional antenna.

\section{B. Smart Antennas Technologie}

Two of the main types of smart antennas include switched beam smart antennas and adaptive array smart antennas. Switched beam systems have several available fixed beam patterns. A decision is made as to which beam to access, at any given point in time, based upon the requirements of the system. Adaptive arrays allow the antenna to steer the beam to any direction of interest while simultaneously nulling interfering signals.

Recent advances in Micro-Electro-Mechanical Systems (MEMS) make possible the realisation of very efficient switch beam antennas (Figure 3). Thanks to theirs mechanical switches, these antennas have a better gain than pure electronic one.

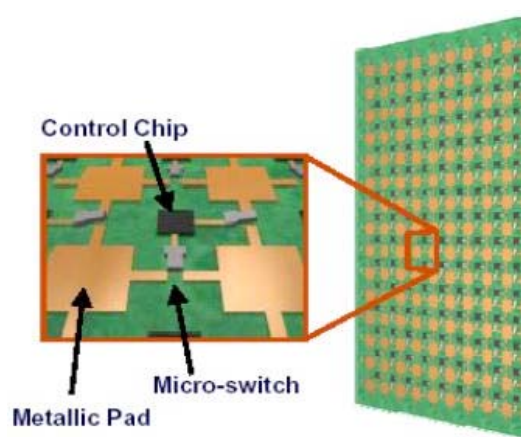

Figure 3. Smart antennae with MEMS switch

In our proposition we consider switch beam antennas. Thus for enabling communication between two nodes, theirs antennas do not need to be aligned, but the right antennae has to be activate.
It adds complexity at the MAC layer and this could be a drawback in the WSN context with scarce computing resources. The following sections propose a simple extension to the 802.15.4 protocol that take advantage of smart antennas with the respect of the resource constraints.

\section{PRELIMINARIES AND OVERVIEW OF MAC PROTOCOLS WITH SMART ANTENNA}

In this section we present the basic concepts used in directional MAC protocols. We present also the most important issues encountered in designing MAC protocols with smart antennas, and some directional MAC protocols cited in the literature. Most of these works is based on 802.11 protocol but is generalizable to other MAC protocols.

\section{A. Basic concepts}

Like the IEEE 802.11, MAC protocols with directional MAC protocols use different mechanisms and control packets in order to coordinate the transmission on the shared wireless medium.

\section{Directional RTS/CTS}

This mode of communication is used in DMAC [4] and in [6] in order to achieve higher communication range with the exploitation of the spatial reusing advantage given by directional antennas. It consists on the transmission of both RTS/CTS and DATA packets in directional mode.

\section{Multiple-Directional RTS/CTS}

This mode consists of the transmission of multiple RTS/CTS packets. Each RTS or CTS packet is transmitted sequentially on each direction to make all the neighbours of the sender and receiver aware of the current communication.

\section{Directional virtual carrier sensing DVCS [3]}

DVCS is an adaptation of the virtual carrier sensing of the IEEE 802.11 in order to support the use of directional antennas. The DVCS permits to the MAC protocol to determine the availability of channel in a specific direction. This concept is implemented through a DNAV (Directionnal Network Allocation Vector) table which is an adaptation of the NAV of the 802.11. Each item of the DNAV table is associated to one direction, and describes for how many time the channel will be occupied in that direction.

\section{Directional allocation vector DNAV}

DNAV (Directional Network Allocation vector) is an adaptation of the NAV used in the 802.11. Each item of the DNAV table is associated to one direction, and describes the state of the channel in that direction (idle, occupied, duration of occupation). The DNAV table is updated on the reception of control or DATA packets.

\section{Cache of Angle of Arrival [3]}

The cache of angle of arrival is used in directional MAC protocols to determine the direction on which a neighbor node is located. When a node receives a packet from its neighbors, it records the angle of arrival (AOA) of the packet and the MAC address of the transmitter in its cache of angle of arrival. This cache of AOA constitutes an 
association between each neighbor and the antenna which permit to transmit a packet to that neighbor.

\section{B. Issues in Designing MAC protocols for wireless networks with directional antenna}

The characteristics of directional antennas as the lack of coordination between mobile nodes makes the design of MAC protocols for directional antennas enabled sensor networks a very challenging task. When a node $\mathrm{A}$ is communicating on a chosen direction, it is not aware of the state of its neighbors located in the other directions. Hence, when it finishes communicating it may attempt to exchange information with another neighbor B. Two scenarios may happen: either the neighbor $\mathrm{B}$ is currently receiving DATA and collision will happen at $\mathrm{B}$, or the node is transmitting and will not be able to respond. To design an efficient directional MAC protocol we must be able to accurately characterize this lack of coordination. We investigate three major coordination problems: the Directional hidden terminal problem, the missing receiver, and deafness problem.

The Directional Hidden terminal problem: the hidden terminal problem is well known in mobile communications. In mobile networks with omnidirectionnal communications, the hidden terminal problem happens when two nodes A and $\mathrm{B}$ attempt to transmit a packet to a common neighbor node C. Transmitted packets collide at the receiver and retransmissions are required.

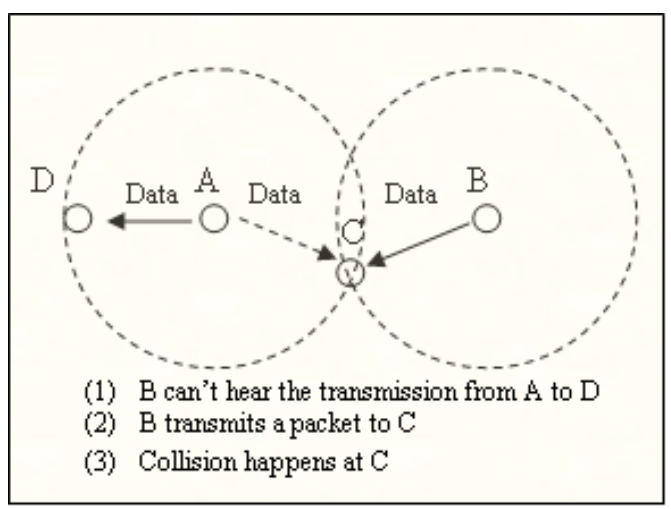

Figure 4. Hidden terminals problem in omni-directional communications

This problem was resolved by the transmission of RTS/CTS control packets prior to data transmission. The goal of these control packets is to inform neighboring nodes about the established communication. With directional antennas this mechanism is not efficient. An instance of this generic problem was identified in [5]. The problem is illustrated in the figure 4 and 5. Suppose that nodes A and B had exchanged RTS/CTS packets and are communicating in directional mode. Since $\mathrm{C}$ is out of the directional range of communication between A and B, it can't hear the communication between $\mathrm{A}$ and $\mathrm{B}$. Now suppose that $\mathrm{C}$ want to initiate a communication with $\mathrm{D}$. $\mathrm{C}$ senses the channel in the direction of $\mathrm{D}$ and concludes that the channel is idle. $\mathrm{C}$ sends an RTS packet to D, and this packet will interfere with packets transmitted from $\mathrm{B}$ to $\mathrm{A}$.

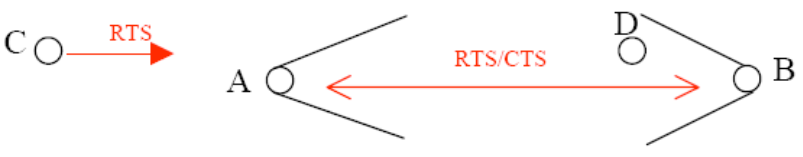

Figure 5. The directional hidden terminals problem The directional hidden terminal problem causes high collision probability when the traffic load increases. An efficient MAC protocol must resolve this problem.

The missing receiver problem: arises when control packets sent on a given direction fails because the receiver node is currently engaged in a directional communication with another node (acting either as transmitter or receiver). To illustrate the problem, we consider the simple three-node scenario of Fig. 6, where node A transmits to node B (flow A-B) and node C transmits to node A (flow C-A). In this example we consider that control packets are transmitted in directional mode.

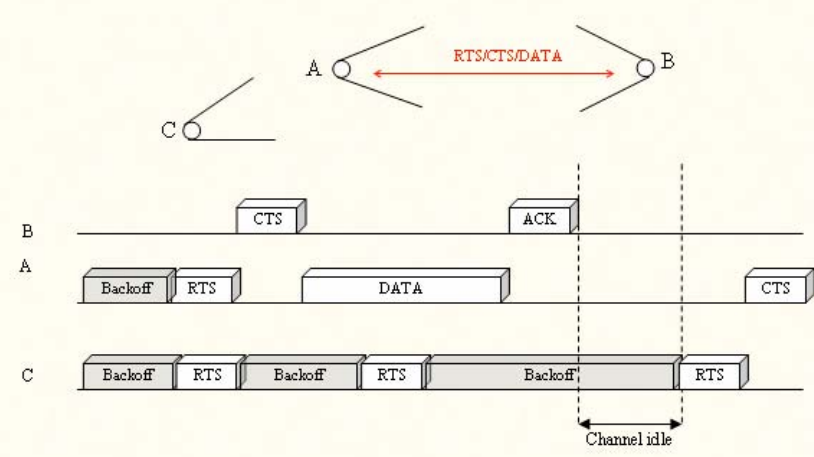

Figure 6: The missing receiver problem

In figure 10 , node $\mathrm{C}$ fails to establish a communication with $\mathrm{A}$ because $\mathrm{A}$ is in directional communication with node $\mathrm{B}$, then node $\mathrm{C}$ will perform a random backoff and retry the process at the expiration of the backoff. Unless proper measures are taken, node $\mathrm{C}$ will enter in backoff phases several times which causes large packet delay for flow C-A and decreases its throughput.

The deafness problem: this problem is a special case of the missing receiver problem. In the case where node $\mathrm{A}$ continuously sends packets to node $B$, these packets have more probability to be transmitted than packets from $\mathrm{C}$, because A will have always a shorter backoff. This process is repeated until the packet is transmitted successfully or up to a maximum number of tries after which the packet is dropped.

The main cause of these three problems is that control packets which are sent in directional mode fail to inform neighboring nodes about the current transmission. An efficient MAC protocol must handle these problems.

Note that the missing receiver and deafness problems do not occur in single-omnidirectionnal wireless networks because $\mathrm{C}$ can carrier sense the data transmissions of $\mathrm{A}$ and immediately defer until the end of A-B transmission. 


\section{Directional MAC protocols}

Several research works [11] have been published in the field of using directional antennas in wireless networks. They either focus on maximizing the special reuse, or on resolving the hidden terminal and deafness problems. Other researches on routing protocols [7] [8] [9] with directional antennas are out of the scope of this article.

Choudhury et al. have proposed the DMAC (directional MAC) protocol [4] which is an adaptation of the IEEE 802.11 standard in order to support directional antennas. DMAC uses the RTS/CTS/DATA/ACK handshake in order to transmit a data packet. DMAC suppose that the upper layer is aware of the position of neighbours so that RTS control packets can be transmitted in directional mode. An idle node listens to the channel in omni-directional mode.

DMAC uses the DVCS mechanism in order to achieve maximum spatial reuse, but it suffers from both deafness and directional hidden terminal problems.

Authors in [4] propose the MMAC protocol which is an extension of the DMAC protocol. MMAC extends the transmission range by establishing directional-directional links (i.e. both the sender and the receiver direct their antennas one in the direction of the other). To do this MMAC uses Multihop-RTS packets which travel hop by hop from the source to the destination. The goal of the Multi-hop-RTS packet is to request the receiver to direct its antenna in the direction of the sender. The MMAC protocol supposes that the upper layer of the source node is able to provide the route from the source to the destination.

The advantage of MMAC is that it permits high range communications which permits to forward packets from the source to the destination in little number of hops. However, like DMAC, MMAC protocol suffers from the directional hidden terminals and deafness problems.

Korakis et al. have proposed the circular-DMAC protocol [2] that tries to address the deafness problem. A node, which has a DATA packet to be transmitted, sends multiple RTS packets directionally in all beams. When the receiver receives the RTS, it replays with a single directional CTS packet. The RTS packets are sent sequentially, so the receiver has to wait to send the CTS until the sender has sent RTS in all its antennas.

\section{D802.15.4}

\section{A. Architecture}

In this paper we propose a novel architecture for wireless sensor networks. The proposed architecture is based on the integration of directional antennas in the physical layer of the IEEE 802.15.4 standard. The main idea of our work is to extend the physical and MAC layer functionalities of the standard in order to support directional antennas. We will call this new model D802.15.4 (for Directional 802.15.4). Figure 7 describes this model.

The physical layer architecture: the IEEE 802.15.4 standard uses a physical layer based on a single omni-directional transceiver. In our architecture we use a directional switched

antenna model composed of four directional antennas and one isotropic antenna.

Each directional antenna is dedicated to one direction and has the transmission and reception capabilities. Also each directional antenna is characterized by its gain of transmission and its gain of reception in a given direction. The isotropic antenna is characterized by its uniform gain of $1 \mathrm{db}$ in all the directions, and is used only for the transmission of broadcast packets. It is used by ZigBee upper layers to transmit commands and beacons.

In order to be compatible with the internet, we maintain the TCP/IP stack of protocols. At the MAC level we adapt the IEEE 802.15.4 in order to support the use of directional antennas at the physical level.

$$
\text { TCPIUDP }
$$
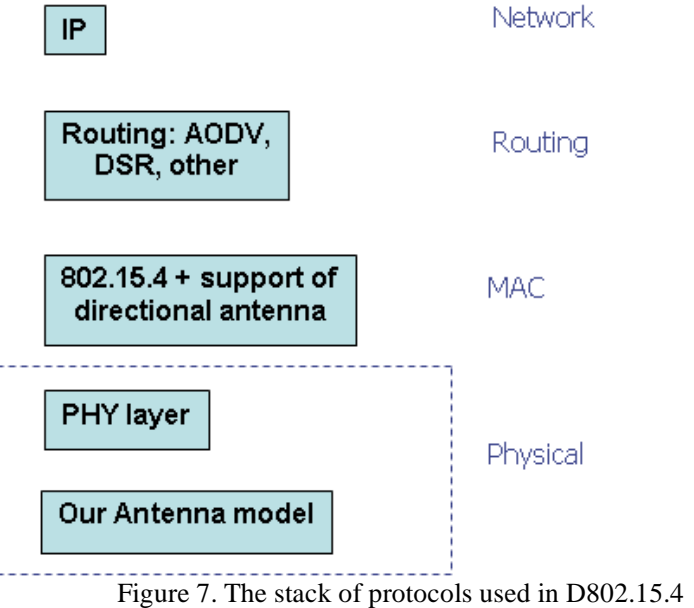

\section{B. The D802.15.4 MAC layer}

The D802.15.4 MAC layer has the following roles:

- Channel access coordination (contention, slotting, CSMA/CA)

- Association and disassociation to the sensor network

- Synchronization between mobile devices

- The switching between the four directional antennas and the isotropic antenna according to the position of the destination node.

- Record the Angle of Arrival of packets in order to use it for the transmission.

In order to record the Angle of Arrival of packets, the MAC layer maintains an Angle of Arrival cache, which contains for each neighbor node the directional antenna to be used in order to send a packet to that node. At the reception of a packet, the MAC layer records the source address of the packet and the antenna by which the packet is received. Each element of the angle of arrival cache has the following fields:

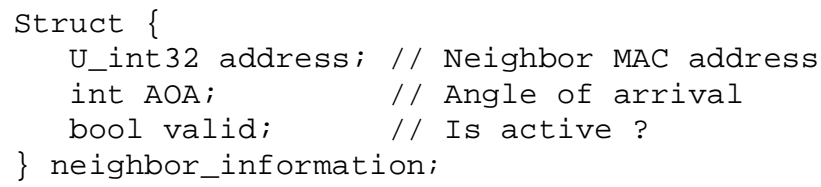

This structure is used by the MAC layer for the transmission of each unicast packet. 
The transmission process: The transmission process is responsible of the switching between antennas and the transmission of DATA packets. When the MAC layer receives a packet $P$ to be transmitted to node $n$, the MAC layer checks if the angle of arrival for the node $n$ had been registered. If the $\mathrm{AOA}$ is registered, the MAC layer requests the physical layer to activate the corresponding antenna and transmit the packet. Else, the MAC layer requests the physical layer to activate the isotropic antenna and to deactivate all the directional antennas. The algorithm of transmission is as follow:

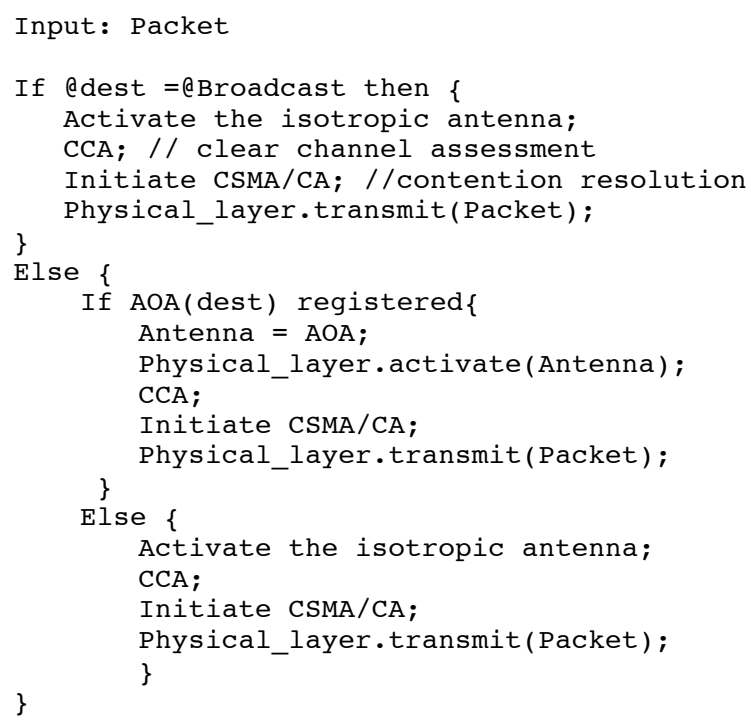

Mobility handling:

In wireless networks nodes are generally mobile. The MAC layer must be able to detect changes of the position of neighbor nodes. In order to have accurate information about the position of neighbor nodes, the MAC layer transmits periodically a Hello packet to announce the position of the mobile node. The AOA of a neighbor node is updated at the reception of Hello packet. The cache of AOA is invalidated by the MAC layer if no Hello packet is received after timeout. In this case only the isotropic antenna is used for the transmission of packets.

\section{Evaluation}

In this section the performance of the D802.15.4 protocol are compared with the IEEE 802.15.4 standard. In order to evaluate the performances of our architecture we use the NS-2 Simulator (Network Simulator 2) [14]. The choice of NS-2 is motivated by its simplicity of utilisation and its modularity.

The 802.15.4 protocol included in the NS-2 base version, has been extended with the previously presented mechanisms. Mainly the Physical and MAC layer have been modified.

\section{A. Study of the effect of topology and traffic patterns}

The impact of the network topologies and the traffic patterns is first evaluated.

\begin{tabular}{|c|c|}
\hline Number of nodes & 25 \\
\hline $\begin{array}{c}\text { Distance between nodes } \\
\text { Transmission range of directional } \\
\text { antennas }\end{array}$ & $10 \mathrm{~m}$ \\
\hline Transmission range of isotropic antenna & $10 \mathrm{~m}$ \\
\hline Main lobe gain & $15 \mathrm{dBi}$ \\
\hline SNR threshold & $6 \mathrm{~dB}$ \\
\hline Reception threshold & $-94 \mathrm{dbm}$ \\
\hline
\end{tabular}

Table 1. Simulation parameters of scenario 1.

\section{Scenario 1: Grid topology}

This scenario is illustrated in Fig. 8 and consists of a grid topology with traffic streams represented by Red arrows. Simulation parameters are described in the table 1 .

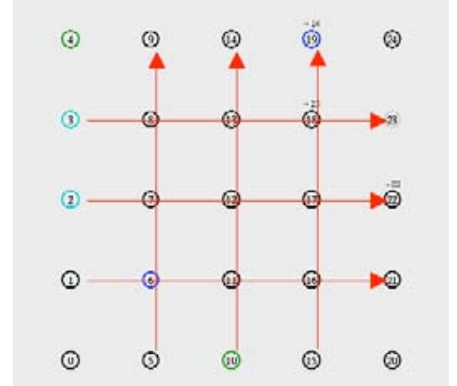

Figure 8 . Network topology of scenario 1

The goal of this scenario is to present the performances of D802.15.4 with linear topologies. We note that these topologies are not the most favourable for D802.15.4 because of the overlapping between the different streams. These scenarios may be present in the case of a sensor network embedded on a train or on a wing of a plane.

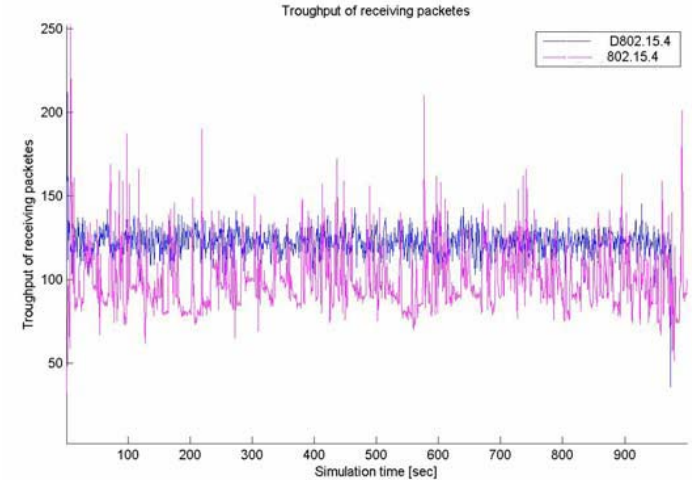

Figure 9. Average throughput versus simulation time for scenario1 


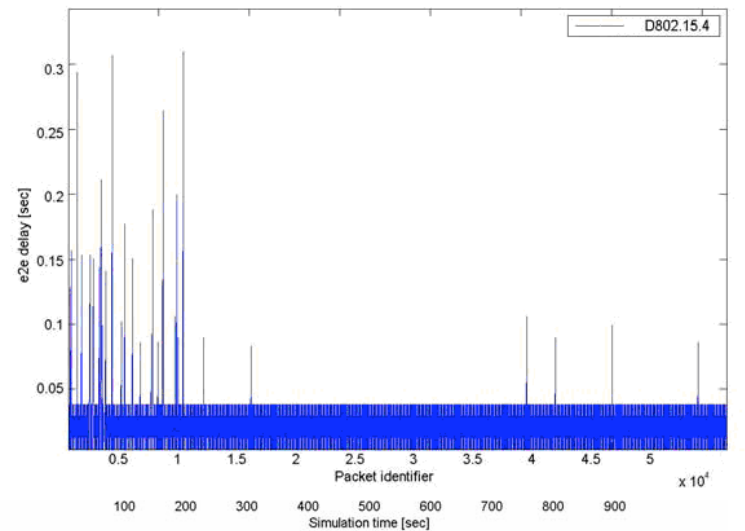

Figure 10. End-to-end delay versus packet identifier for D802.15.4

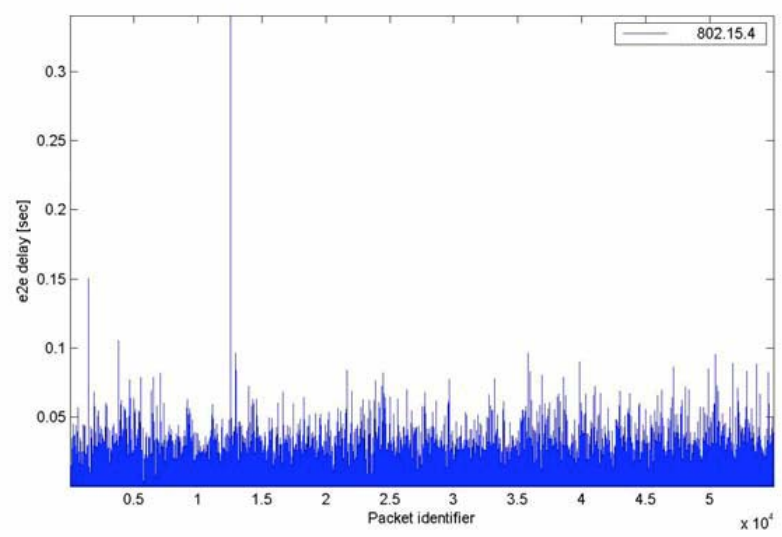

Figure 11. End-to-end delay versus packet identifier for 802.15.4

Fig.10 and fig.11 depict the end-to-end delay versus packet identifier of D802.15.4 and IEEE 802.15.4 protocols respectively. Fig.9 depicts the throughput of receiving packets. For this scenario, similar performances of delay and throughput between the D802.15.4 and the IEEE 802.15.4 protocols are observed. However the delay is more stable in D802.15.4 because of the lower rate of collision in the D802.15.4. This variation of the end-to-end delay is called jitter. This parameter is an important metric in performance evaluation and especially for real-time applications. Simulation results have shown that unlike the IEEE 802.15.4 standard, the D802.15.4 permits to minimise the jitter and delay variation.

\section{Scenarios 2 \& 3: Crossing Streams}

In the two following scenarios $(2 \& 3)$ the topology linearity has been decreased to avoid the overlapping situations. The same parameters of simulation as scenario 1 have been used. The network topology of scenario 3 consists of 25 mobile nodes randomly deployed on $80 \times 80$ meters area. The topology and the streams are illustrated in figure $12 \& 13$.

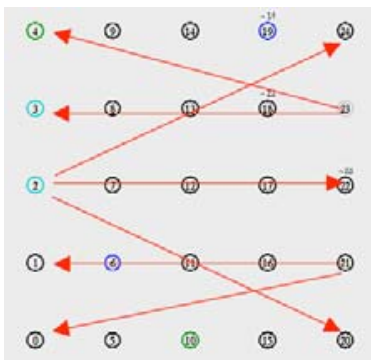

$\begin{array}{ll}\text { a) scenario } 2 & \text { b) scenario } 3\end{array}$

b) scenario 3

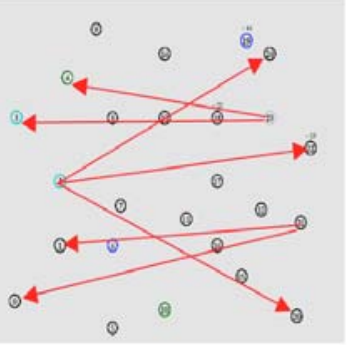

scenario 2 \& 3

Fig.14 and Fig.15 depict the end-to-end delay of the D802.15.4 and the IEEE 802.15.4 protocols respectively in scenario 2 .

Figure 13: Throughput of receiving packets in scenario 2

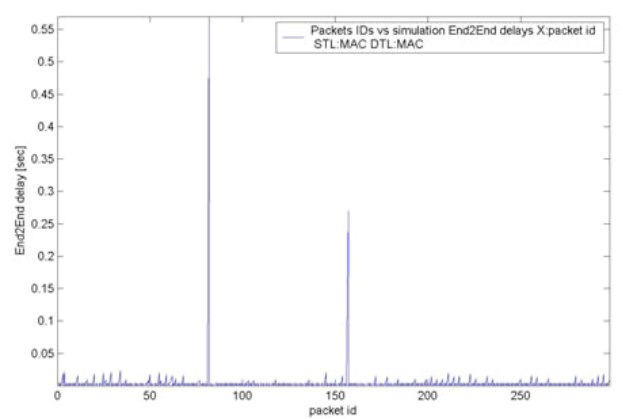

Figure 14: end-to-end delay versus packet identifier for D802.15.4 in scenario 2

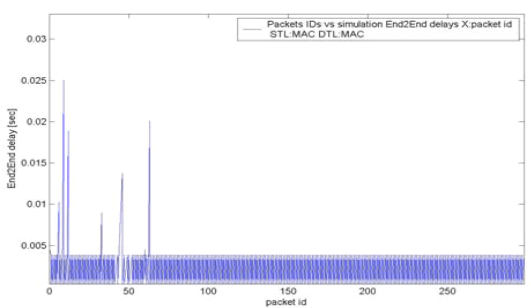

Figure 15: end-to-end delay versus packet identifier for IEEE 802.15.4 in scenario 2

Fig.16 shows the aggregate throughput in packets/sec for the three considered protocols with the same traffic patterns in the third scenario. 


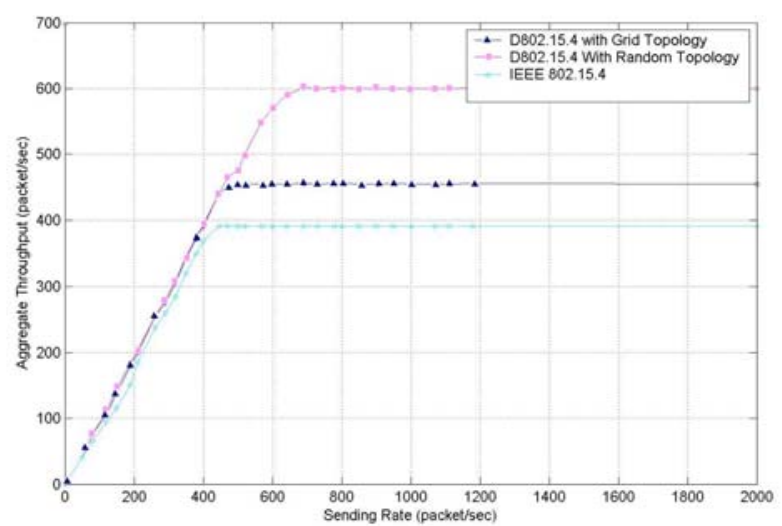

Figure 16. Aggregate throughput of D802.15.4 with random topology, D802.15.4 with grid topology and IEEE 802.15.4

The results of these scenarios confirm our assumption about the impact of the topology on the performances of the D802.15.4 protocol.

The average throughput of the protocol D802.15.4 had been increased by $30 \%$ when applied to a topology with crossing streams. This is due to the reduction of the number of collisions between nodes because of the absence of overlapping between flows.

As example, the transmission between nodes 2 and 24 through node 13 in figure 12 will not interfere with other transmissions in the network. Each stream uses a transmission space which is independent of the transmission spaces used by other flows.

This study has shown that the performances of D802.15.4 depend strongly on the network topology and traffic patterns. Networks which have the form of grids are the most unfavourable for D802.15.4. Traffic patterns have also an impact on performance. The linearity of traffic flows contributes drastically in the degradation of performances of our protocol. On the other hand our protocol has proven its efficiency in random topology networks with linearly independent traffic flows.

\section{B. Study of the effect of distance between nodes}

In this section we analyse the impact of the distance between nodes on the performances of the D802.15.4 protocol. 12 scenarios are studied; in each scenario we randomly deploy the nodes on the simulation area. Each scenario is characterized by the distance between nodes. The simulation parameters are illustrated in the following table:

\begin{tabular}{|c|c|}
\hline Number of nodes & 100 \\
\hline Coverage area & $80 \times 80 \mathrm{~m}$ \\
\hline Transmission range of directional antennas & $35 \mathrm{~m}$ \\
\hline Transmission range of isotropic antenna & $15 \mathrm{~m}$ \\
\hline Main lobe gain & $10 \mathrm{~dB}$ \\
\hline SNR threshold & $6 \mathrm{~dB}$ \\
\hline Reception threshold & $-94 \mathrm{dbm}$ \\
\hline
\end{tabular}

Table 2: Simulation parameters used for the study of the effect of distance between nodes.

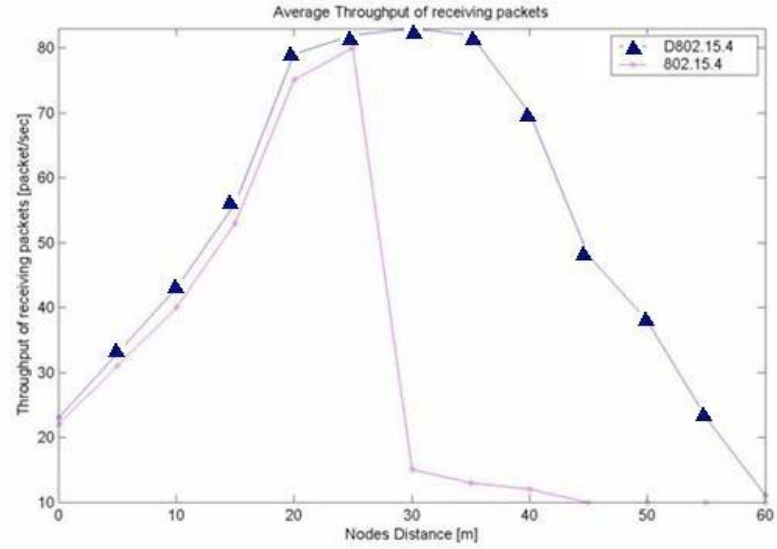

Figure 17: average throughput versus distance between nodes for the 802.15.4 and D802.15.4 protocols.

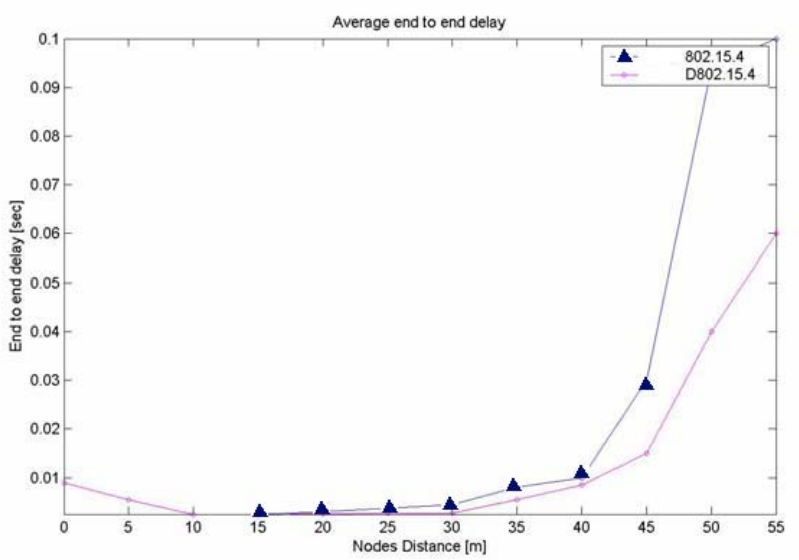

Figure 18: end-to-end delay versus distance between nodes for the 802.15.4 and D802.15.4 protocols.

Fig.17 illustrates that the D802.15.4 and the 802.15.4 protocols have similar performances as long as the distance between nodes is less than 30 meter. Beyond that distance, the D802.15.4 protocol outperforms the 802.15.4 Protocol. The figure illustrates that the throughput of the 802.15.4 decreases drastically when the distance between nodes is greater then 30 meter. This is explained by the advantage of the gain of directional antennas in D802.15.4. The gain of directional antennas permits a higher communication range in transmission and reception which permits to convey packets in less number of hopes.

Fig.18 illustrates the delay obtained by the two protocols. The end-to-end delay achieved by the 802.15.4 and D802.15.4 are similar as long as the distance between nodes is less than 35 meter. Beyond that distance the delay of the 802.15.4 increases exponentially.

This study has illustrated the advantages of the D802.15.4 protocol with reference to the IEEE 802.15.4 standard. Indeed thanks to its concept of gain, the D802.15.4 protocol permits to realize a higher communication range. This permits to extend the coverage area of the network with a reduced number of nodes. The limited communication range of the 802.15.4 contributes in limiting the coverage area as illustrated in Fig.17. By the way the D802.15.4 protocol is more adapted to applications which require long distance 
between mobile nodes. An application which requires $n$ mobile node in order to cover an area $\mathrm{Z}$ with the 802.15.4 protocol, requires a number of nodes $n^{\prime}<<n$ with the D802.15.4 to cover the same area.

\section{CONCLUSION}

This paper has proposed an evolution of the 802.15.4 protocol to take benefits of smart antennas. Simulations have shown the benefits and the feasibility of such architecture for wireless sensor networks.

However, the coming 802.15.4A protocol based on recent advances in wireless transmission techniques promises good opportunities for this architecture. Indeed, the MAC layer is quite similar to the first version of the 802.15.4 protocol, but the physical layer uses the millimeter wave band. Then, as the antennas are smaller and more directive, the building of very small antennas switches will be made easier. Moreover, the use of IR-UWB techniques reduces the energy consumption and makes simplest localization algorithms, two goals that can be improve using smart antennas.

\section{REFERENCES}

[1] Saad Alabeedi, "Antenna Beamforrming for ad hoc networks", course of Carleton University.

[2] T.Korakis, G.Jakllari, L.Tassiulas, "A MAC protocol for full exploitation of directional antennas in AD-hoc Wirless networks" Proc. MobiHoc 2003, pp. 98-107.

[3] M. Takai, J.Martni, A.Ren, R. Bagrodia, "Directionnal virtual carrier sensing for directional antennas in mobile Ad hoc networks". In Proceedings of the 3rd ACM international symposium on Mobile ad hoc networking and computing, pages 183-193. ACM Press, 2002.

[4] R.R.Choudhury, X.Yang, R.Ramanathan, N.Vaidya, "Using directional antennas for Medium acces control in Ad Hoc networks" MOBICOM'02, September 23-28, 2002, Atlanta, Georgia, USA.

[5] R.R.Choudhury, N.Vaidya, "Deafness: A MAC problem in Ad Hoc networks when using directional antennas". Technical report, University of Illinois at Urbana-Champaign, July 2003.

[6] R.Ramanathan, " Ad Hoc networks with directional antennas: A complete system solution". IEEE JOURNAL ON SELECTED AREAS IN COMMUNICATIONS, VOL. 23, NO. 3, MARCH 2005

[7] C.E.Perkins, E.M. Royer, "Ad-hoc On-Demand Distance Vector Routing" Proceedings of the 2nd IEEE Workshop on Mobile Computing Systems and Applications, New Orleans, LA, February 1999, pp. 90-100.

[8] A.Nasipuri, J.Mandava, H.Manchala, R.E.Heromoto, "On-Demand Routing Using Directional Antennas in Mobile Ad Hoc Networks" Computer Communications and Networks, 2000. Proceedings. Ninth International Conference on 16-18 Oct. 2000 Page(s):535 - 541

[9] R.R.Choudhury, N.Vaidya, "Impact of Directional Antennas on Ad Hoc Routing". Proceedings of 8th Conference on Personal and Wireless Communication (PWC), 2003.

[10] M. Takai, J.Zhou, R.Bagrodia, "Adaptive Range Control Using Directional Antennas in Mobile Ad-Hoc Networks" MSWiM'03, September 19, 2003, San Diego, California, USA.

[11] R.Ramanathan, " On the Performance of Ad Hoc Networks with Beamforming Antennas”. In proceedings of MobiHoc,pp. 95 - 105, October 2001.

[12] Specifications for Low-Rate Wireless Personal Area Networks. http://standards.ieee.org/getieee802/download/802.15.4-2003.pdf

[13] Zigbee Specification. http://www.zigbee.org/en/index.asp

[14] Network Simulator home page. www.isi.edu/nsnam/ns 